\title{
Commentary: Cortical responses to salient nociceptive and not nociceptive stimuli in vegetative and minimal conscious state
}

\author{
Antonino Naro and Rocco S. Calabrò * \\ Behavioral and Robotic Neurorehabilitation Laboratory, IRCCS Centro Neurolesi "Bonino-Pulejo," Messina, Italy
}

Keywords: pain perception, LEP, DOC, VS, MCS

\section{A commentary on}

Cortical responses to salient nociceptive and not nociceptive stimuli in vegetative and minimal conscious state

by de Tommaso, M., Navarro, J., Lanzillotti, C., Ricci, K., Buonocunto, F., Livrea, P., et al. (2015). Front. Hum. Neurosci. 9:17. doi: 10.3389/fnhum.2015.00017

We read with great interest the work by de Tommaso et al. (2015) assessing cortical responsiveness to nociceptive and multimodal sensory stimuli in patients affected by chronic disorders of consciousness (DOC). In their valuable work, the authors attempted to assign each patient to the most appropriate DOC category by using auditory, visual, somatosensory, and nociceptive laser

OPEN ACCESS

Edited by:

Marta Olivetti,

Sapienza University of Rome, Italy

Reviewed by:

Naofumi Otsuru,

Hiroshima University, Japan

André Mouraux,

Université Catholique de Louvain,

Belgium

${ }^{*}$ Correspondence:

Rocco S. Calabrò

salbro77@tiscali.it

Received: 24 August 2015 Accepted: 19 November 2015 Published: 08 December 2015

Citation:

Naro A and Calabrò RS (2015) Commentary: Cortical responses to

salient nociceptive and not nociceptive stimuli in vegetative and minimal conscious state.

Front. Hum. Neurosci. 9:657. doi: 10.3389/fnhum.2015.00657 stimuli. Interestingly, the motor responsiveness to nociceptive stimuli assessed through the Coma Recovery Scale-Revised and the Nociception Coma Scale-Revised correlated with each evoked response, but Laser Evoked Potentials (LEPs). The latter were recognizable in all of the patients, independently from the preservation of other sensory modalities and the motor responsiveness to nociceptive stimuli. Hence, LEP absence should not be considered as a demonstration of the inability to experience pain, since the preservation does not per se indicate a conscious pain perception in DOC (de Tommaso et al., 2015). However, it is to note that we should be aware in interpreting authors' findings since these are based on a very limited number of patients (i.e., only 4 MCS and 5 VS). Moreover, since the presence vs. absence of LEPs was based on a subjective visual identification of the event-related potential waveforms, the possibility of misinterpretations (mainly due to very low signal-to-noise ratio) should be taken into account.

Conscious pain perception in DOC patients is a very thorny matter of ethical and clinical debate. Indeed, it is not easy to clinically assess pain perception in the vegetative state (VS) (characterized by non-conscious reflexive behavioral patterns) and minimally conscious state (MCS) patients (characterized by reproducible but fluctuant conscious behavioral patterns), although several clinical scales have been ad hoc employed to improve diagnostic accuracy (Chatelle et al., 2012). In addition, functional neuroimaging studies have shown that some DOC individuals can show residual complex brain activations that do not correspond to their behavioral output (e.g., Kassubek et al., 2003; Boly et al., 2008). Thus, a potential pain experience should be considered even in VS individuals, independently from their communication skills.

Although LEPs are commonly used when studying nociceptive pathways, their role concerning pain assessment in DOC individuals could be at first glance limited by the following issues: (i) the basic LEP parameters, including amplitude and latency, cannot give unique information concerning conscious pain perception in DOC patients (de Tommaso et al., 2015); (ii) LEPs mainly 
constitute a marker of relevant-stimulus dependent arousal (Mouraux and Iannetti, 2009); (iii) LEPs depend on the selective activation of thermo-nociceptive afferents; and (iv) LEP reflect the activity of multiple cortical assemblies within different cortical areas that process either nociceptive or somatosensory inputs (Garcia-Larrea et al., 2003). Nevertheless, the presence or the absence of LEPs could be somehow useful in determining whether or not a patient has the ability to experience pain, although LEPs are not considered a specific marker of pain perception. To this end, some correlations between perceived pain intensity and single-LEP features have been documented (Iannetti et al., 2005; Huang et al., 2013).

In our opinion, further LEP protocols should be fostered in an attempt to more reliably assess pain-perception in DOC patients and reach a more reliable differential diagnosis. In fact, recent studies in healthy individuals have suggested that LEPrelated $\gamma$-band oscillatory activity (GBO) may have a role in modulating pain intensity within primary somatosensory cortex

\section{REFERENCES}

Boly, M., Faymonville, M. E., Schnakers, C., Peigneux, P., Lambermont, B., Phillips, C., et al. (2008). Perception of pain in the minimally conscious state with PET activation: an observational study. Lancet Neurol. 7, 1013-1020. doi: 10.1016/S1474-4422(08)70219-9

Chatelle, C., Majerus, S., Whyte, J., Laureys, S., and Schnakers, C. (2012). A sensitive scale to assess nociceptive pain in patients with disorders of consciousness. J. Neurol. Neurosurg. Psychiatry 83, 1233-1237. doi: 10.1136/jnnp-2012-302987

de Tommaso, M., Navarro, J., Lanzillotti, C., Ricci, K., Buonocunto, F., Livrea, P., et al. (2015). Cortical responses to salient nociceptive and not nociceptive stimuli in vegetative and minimal conscious state. Front. Hum. Neurosci. 9:17. doi: 10.3389/fnhum.2015.00017

Garcia-Larrea, L., Frot, M., and Valeriani, M. (2003). Brain generators of laserevoked potentials: from dipoles to functional significance. Neurophysiol. Clin. 33, 279-292. doi: 10.1016/j.neucli.2003.10.008

Gross, J., Schnitzler, A., Timmermann, L., and Ploner, M. (2007). Gamma oscillations in human primary somatosensory cortex reflect pain perception. PLoS Biol. 5:e133. doi: 10.1371/journal.pbio. 0050133

Huang, G., Xiao, P., Hu, L., Hung, Y. S., and Zhang, Z. (2013). Singletrial laser-evoked potentials feature extraction for prediction of pain perception. Conf. Proc. IEEE Eng. Med. Biol. Soc. 2013, 4207-4210. doi: 10.1109/embc.2013.6610473

Iannetti, G. D., Zambreanu, L., Cruccu, G., and Tracey, I. (2005). Operculoinsular Cortex encodes pain intensity at the earliest stages of cortical processing as indicated by amplitude of laser-evoked potentials in humans. Neuroscience 131, 199-208. doi: 10.1016/j.neuroscience.2004. 10.035
(Gross et al., 2007; Zhang et al., 2012). It has been recently showed that fronto-parietal GBO and the associated sensorymotor integration processes may reflect the functionality of the fronto-cingulate-parietal network involved in pain perception and pain-gating processes at cortical level, independently from the patient's ability to communicate (Naro et al., 2015a,b).

Unfortunately, GBO analysis presents some limiting factors, including low signal-to-noise ratio, high inter-individual variability, and contamination of ocular movements and/or muscle artifacts. Nonetheless, the evaluation of LEP-related GBO might contribute to uncover residual pain perception even in those totally non-communicative patients, even though it is still debated whether or not GBO can be reliably assessed at single-subject level.

Future studies should be fostered in an attempt to shed some light on the role of combined LEP-GBO analysis in pain perception assessment in DOC patients.
Kassubek, J., Juengling, F. D., Els, T., Spreer, J., Herpers, M., Krause, T., et al. (2003). Activation of a residual cortical network during painful stimulation in long-term post-anoxic vegetative state: a ${ }^{15} \mathrm{O}-\mathrm{H}_{2} \mathrm{O}$ PET study. J. Neurol. Sci. 212, 85-91. doi: 10.1016/S0022-510X(03)00106-0

Mouraux, A., and Iannetti, G. D. (2009). Nociceptive laser-evoked brain potentials do not reflect nociceptive-specific neural activity. J. Neurophysiol. 101, 3258-3269. doi: 10.1152/jn.91181.2008

Naro, A., Leo, A., Bramanti, P., and Calabrò, R. S. (2015a). Moving towards conscious pain processing detection in chronic disorder of consciousness: the Anterior Cingulate Cortex neuromodulation. J. Pain. 16, 1022-1031. doi: 10.1016/j.jpain.2015.06.014

Naro, A., Leo, A., Russo, M., Quartarone, A., Bramanti, P., and Calabrò, R. S. (2015b). Shaping thalamo-cortical plasticity: a marker of cortical pain integration in patients with post-anoxic unresponsive wakefulness syndrome? Brain Stimul. 8, 97-104. doi: 10.1016/j.brs.2014.09.001

Zhang, Z. G., Hu, L., Hung, Y. S., Mouraux, A., and Iannetti, G. D. (2012). Gamma-band oscillations in the primary somatosensory cortex: a direct and obligatory correlate of subjective pain intensity. J. Neurosci. 32, 7429-7438. doi: 10.1523/JNEUROSCI.5877-11.2012

Conflict of Interest Statement: The authors declare that the research was conducted in the absence of any commercial or financial relationships that could be construed as a potential conflict of interest.

Copyright (c) 2015 Naro and Calabrò. This is an open-access article distributed under the terms of the Creative Commons Attribution License (CC BY). The use, distribution or reproduction in other forums is permitted, provided the original author(s) or licensor are credited and that the original publication in this journal is cited, in accordance with accepted academic practice. No use, distribution or reproduction is permitted which does not comply with these terms. 